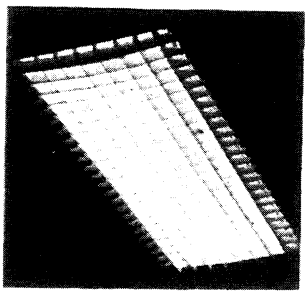

\title{
Iluminación artificial
}

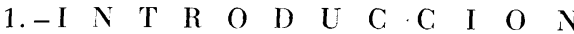

1. OÑtTE, Ingeniero de Caminos

2. - PLANTEAMIENTO DEL PROBLEMA

3. - A I U I B R A I) () E X T E R I O R

4. - A I U M B A D O I N T E R I O R

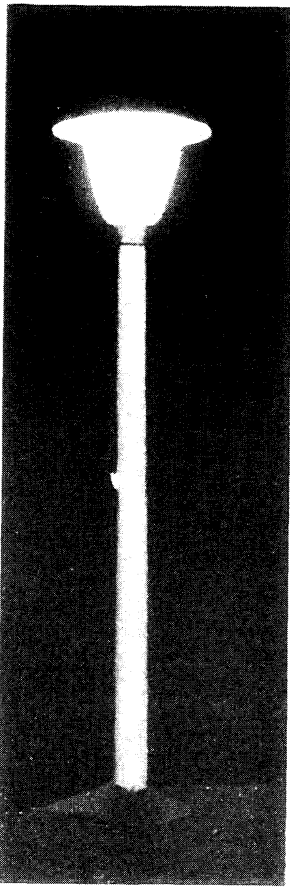

$352 \cdot 4$

\section{SINOPSIS}

Se describen las instalaciones de alumbrado, tanto exterior como interior, indicando las bases de partida que hail servido para proyectarlas.

INFORMES DE LA CONSTRUCCION OI

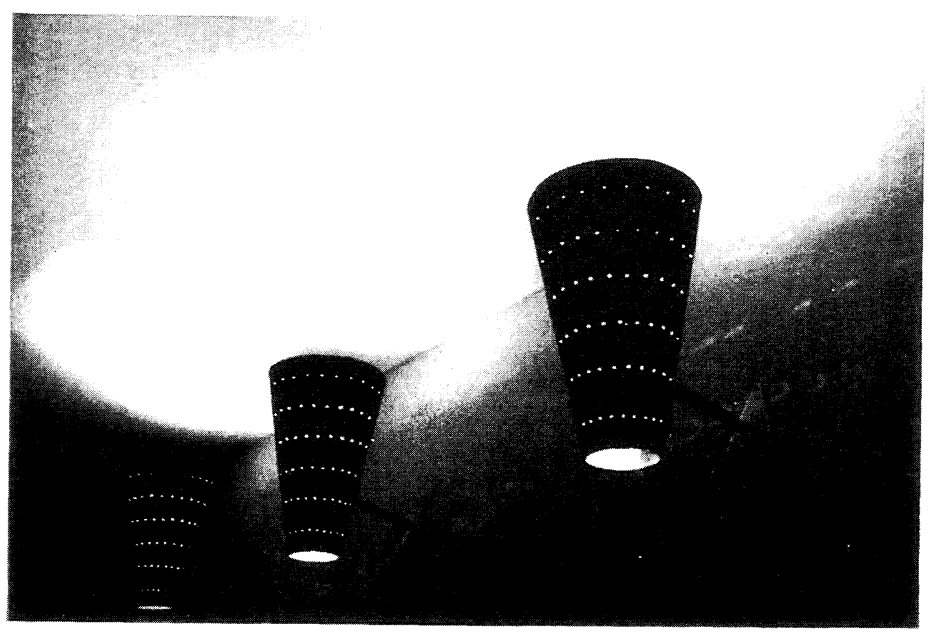




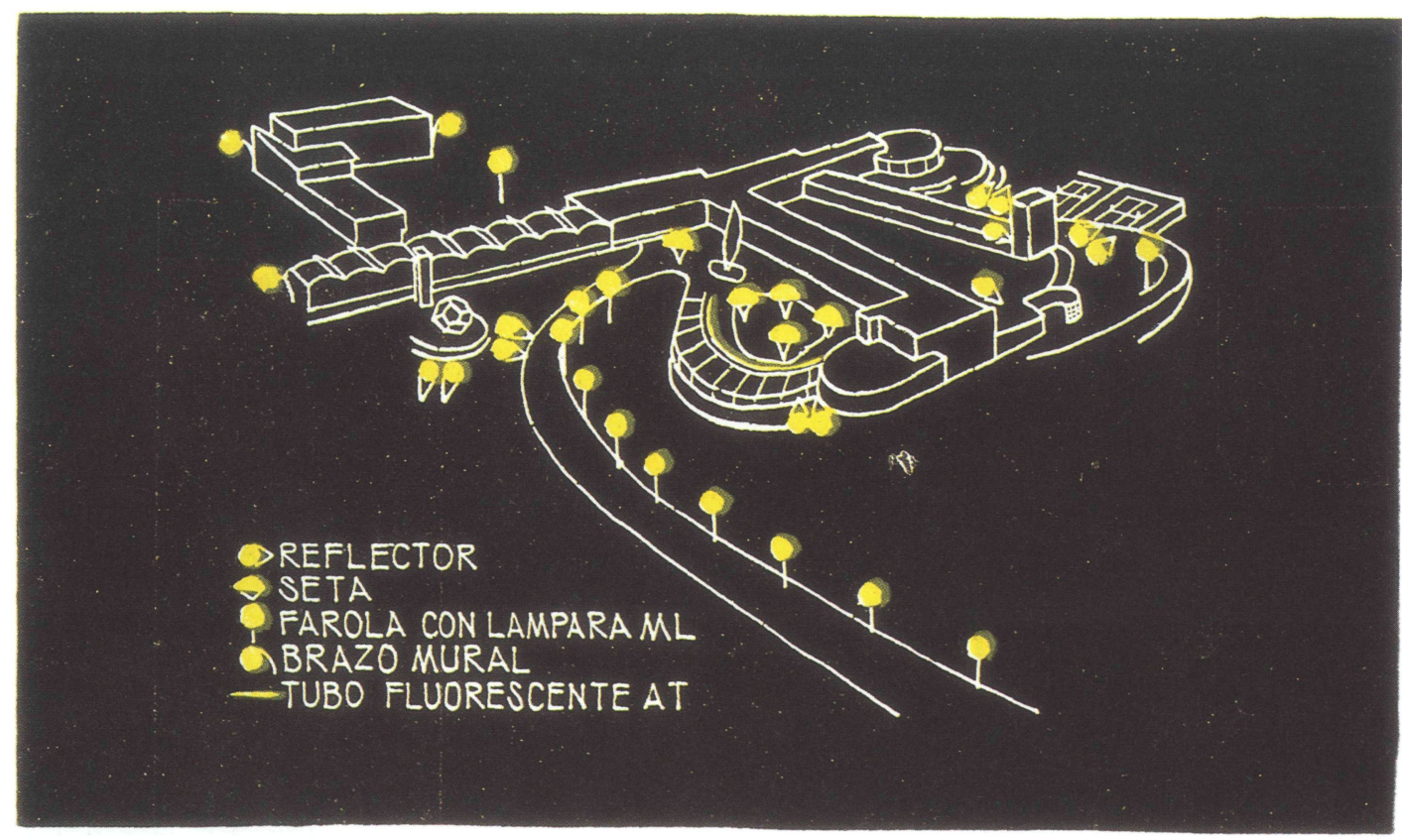

\section{I. - INTRODUCCION}

E1 alumbrado artificial desempeña un papel de considerable importancia entre las instalaciones de un edificio.

Nada más lejos de nuestra intención, al escribir lo que antecede, que comenzar estas líneas con el conocido "Importancia de la asignatura" de los textos escolares de una época no muy lejana todavía, o ensalzar la trascendencia del alumbrado en menoscabo del papel a desempeñar por otras instalaciones anejas a un edificio.

Unicamente pretendemos expresar lo que constituve una grave preocupación para el provectista del alumbrado relatito a la obra de un arquitecto.

La creación arquitectónica, para merecer tal nombre, ha de estar informada en unos conceptos estéticos que obligan a perseguir resultados de trascendencia mucho mavor que la defensa de la intemperie o el sostenimiento de sobrecargas a una determinala altura sobre el terreno. Quertmos decir con esto, que la creación arquitectónica debe ser bella.

Refiriéndonos al caso concreto de un edificio, diremos que su belleza se refiere tanto a su aspecto exterior como al interior.

La exterior se consigue por medio de una ar. tística distribución de líneas, planos y volúmenes y se percibe exclusivamente a través del sentilo de la rista. La luz es, por tanto, el vehiculo que lleva hasta los observadores el mensaje de belleza creado por el arquitecto. Sir luz no hay creación arquitectónica, puesto que no es posible la sensación estética.

De día, el sol, $r$ de noche, el alumbrado artificial. hacen físicamente posible la creación arquitectónica que, de otro modo, no sería realizable por falta de espectadores.

La belleza interior se refleja en la impresión psicológica que reciben las personas que hacen uso del edificio. Aquí conviene observar que, a diferencia de lo que ocurría en el caso de los exteriores, tal impresión no se percibe únicamente a través del sentido de la vista, sino aue puede rerse influída por otros factores como la temperatura ambiente, la rentilación, las condiciones acústicas, etc,

Es interesante señalar que estos factores no desempeñan ningún papel en el valor estético exterior de un edificio, porque el observador, por una operación inconsciente, no atribuye al arquitecto sus sensaciones de frío o calor, etc., cuando está a la intemperie contemplando un edificio.

Dejando a un lado estas últimas ideas que, aunque sugestiras, pudieran desviarnos notablemente de nuestro objetivo, repetiremos lo dicho más arriba: al provectar la instalación de alumbrado artificial debe sentirse la preocupación de rivada del hecho de que, durante determinadas 
horas, el valor estético del edificio ha de percibirse a través de la luz.

Por esto decíamos que el alumbrado artificiai desempeña un papel de considerable importancia entre las instalaciones de un edificio. La importancia es tanto mayor cuanto lo sea la categcría del inmueble.

Y ¿̇en qué consistirá el acierto del alumbrado artificial? Precisamente en que no desvirtúe la personalidad del edificio. En que, bajo la luz artificial, el aspecto del inmueble se conserve dentro del espíritu que le infundió el arquitecto.

Una vez hechos los anteriores comentarios, que consideramos necesarios para la acertada interpretación de lo que sigue, nos enfrentaremos con el tema que hemos venido a considerar en esta ocasión: el alumbrado artificial del nuevo edificio del Instituto en los pinares de Chamartín o, designándolo por su nombre propio, de COSTILLARES.

\section{II. -PLANTEAMIENTO DEL PROBLEMA}

E1 conjunto de edificios que constituven las nuevas instalaciones del Instituto Técnico de la Construcción y del Cemento, ofrece una serie de características arquitectónicas $y$ constructivas que le comunican una acusada personalidad.

Tales particularidades fueron consideradas en el momento de estudiar las instalaciones de alumbrado y sirvieron para plantear los puntos de partida del estudio de las soluciones.

En la instalación de que nos ocupamos consideramos dos partes: el alumbrado exterior y el interior.

Para establecer los datos del problema y descubrir los rasgos fundamentales de las dos instalaciones que habíamos de provectar, observamos el edificio desde el punto de vista de cada una de ellas. Es decir, que intentamos establecer cuáles eran las características arquitectónicas que debían ser tenidas en cuenta al diseñar las instalaciones interior y exterior. $\mathrm{O}$, en otras palabras, rimos de extraer del edificio sus notas características para observadores situados en el exterior y para los ocupantes de sus dependencias.
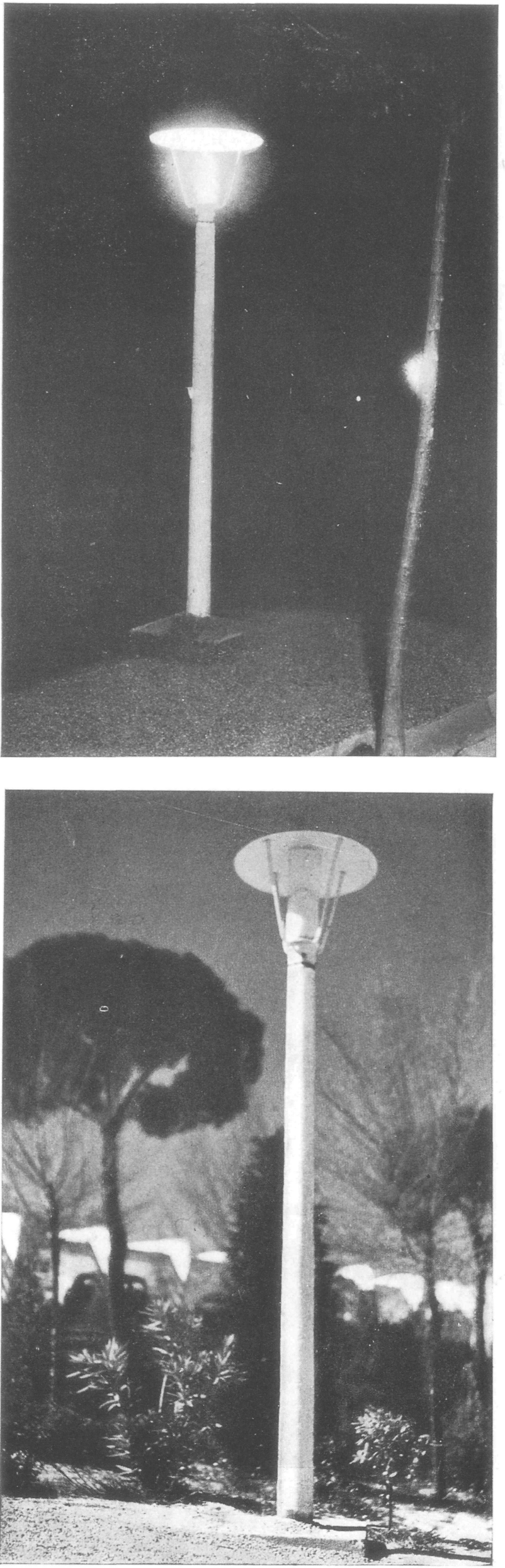

http://informesdelaconstruccion.revistas.csic.es 


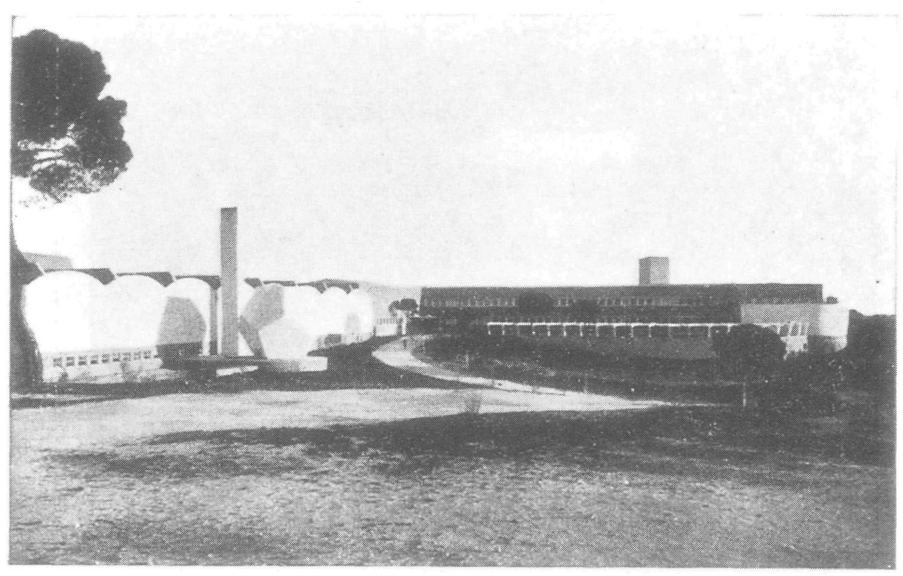

Fig. 4

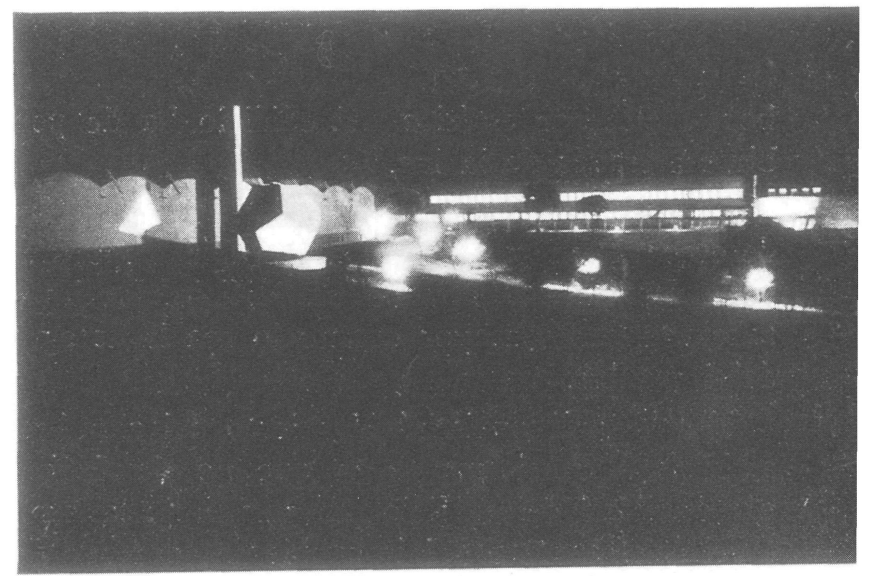

Fig. 5
Nuestras conclusiones fueron las siguientes:

En el exterior se trata de un edificio sobrio, bajo, de perfiles estilizados y en el que predomina la línea recta sobre la curva y la horizontal

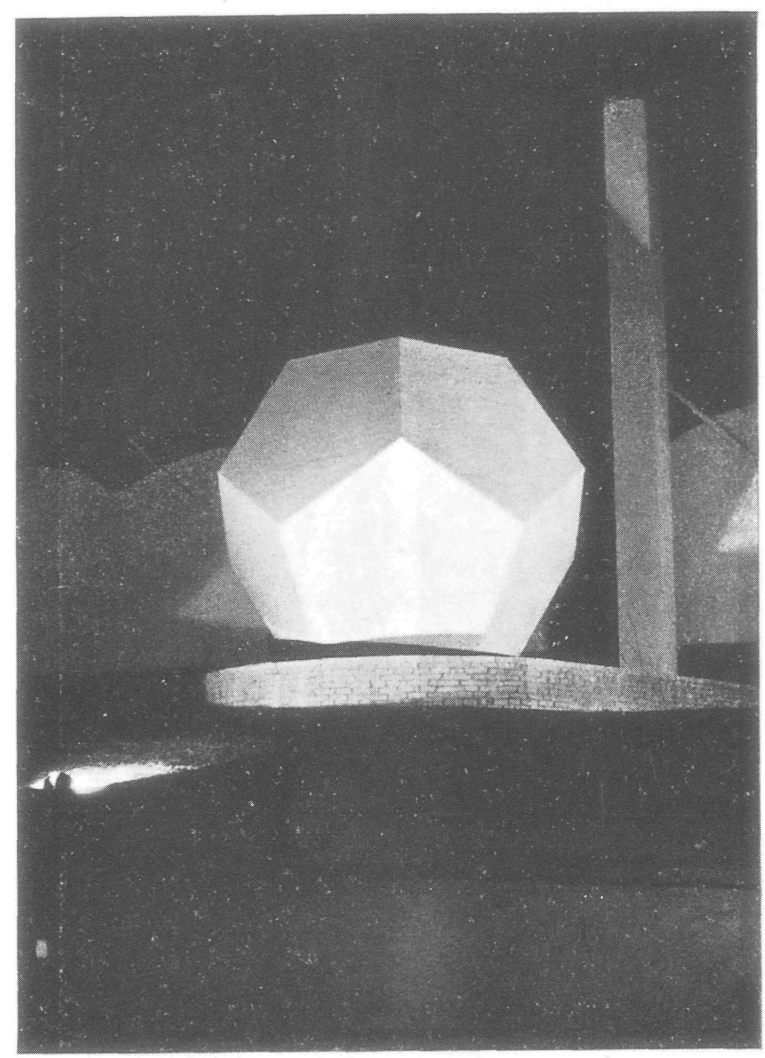

Fig. 6 sobre la vertical. Salvo las bóvedas de la zona de talleres, el silo de carbón, la chimenea de la sala de calderas y la torre del depósito elevado, y aun éstos con la clara finalidad de quebrar la monotonía, todos los elementos de la composición están limitados por líneas horizontales.

Los puntos más llamativos son, precisamente, estas excepciones.

En lo que a colores se refiere, los más abundantes y característicos son el rojo del ladrillo Y el blanco de la piedra artificial, revocos y enlucidos. El verde de la regetación tiene una apreciable participación en el conjunto.

Interiormente, nos encontrames con un edificio de gran austeridad, de generosa iluminación natural $\mathrm{y}$ de colores sumamente claros. Unicamente es oscuro el parimento de los pasillos, vestíbulos y escaleras.

En el edificio principal distinguimos tres zonas claramente diferenciadas:

a) La nave de talleres y ensayos mecánicos, de ambiente industrial.

b) Las naves de estudios y laboratorios, oficinas, despachos y salas de física y química.

c) La nave de servicios generales, con el comedor, salas de recreo, guardarropas, capilla, etc., etc.

El edificio de hormigones, salvo algunos despachos, corresponde todo él al tipo industrial.

Del conjunto se destacan la zona de Dirección, la sala de conferencias, el hall principal, y la entrada y hall de personal. 


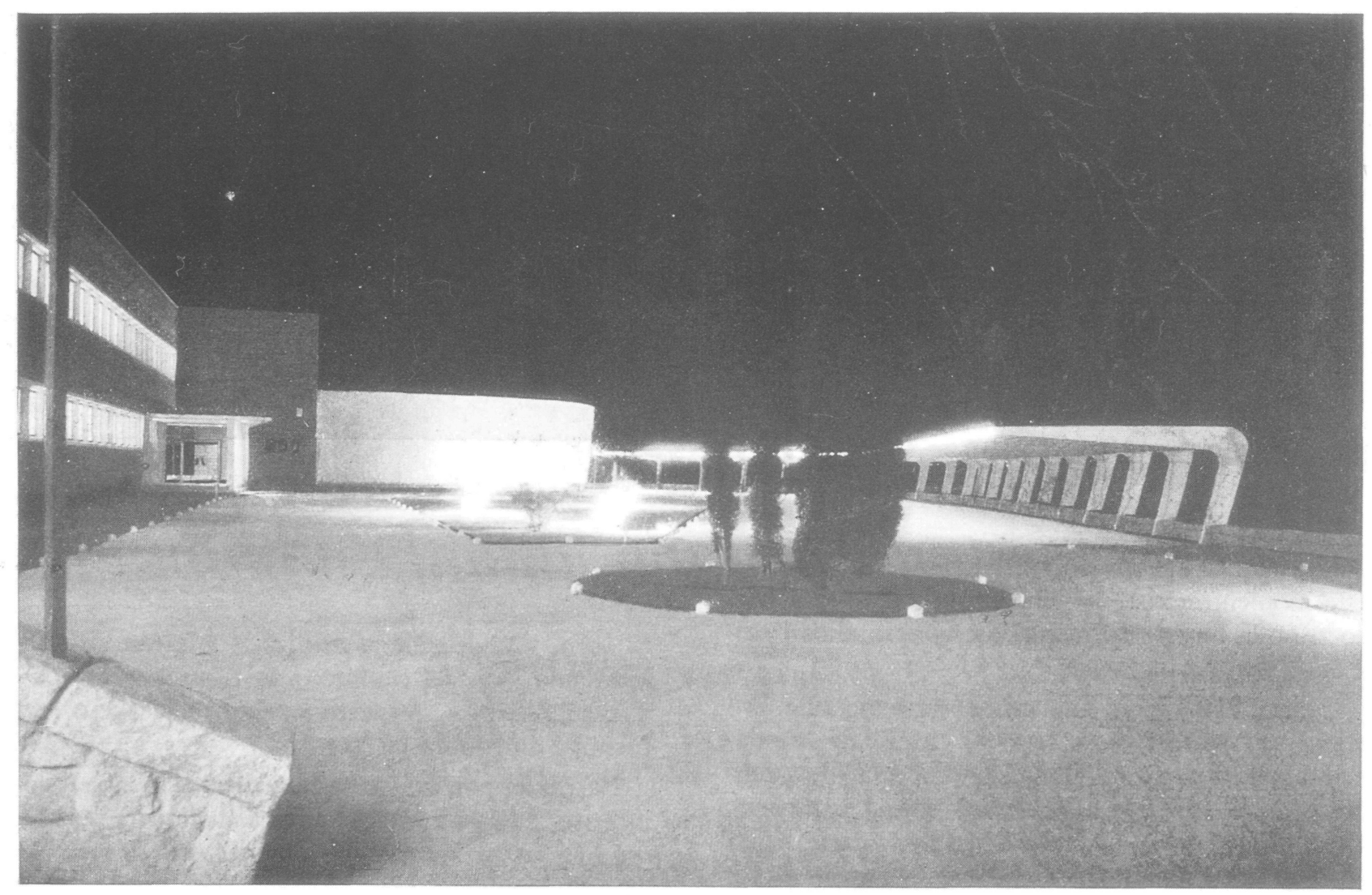

Fig 7

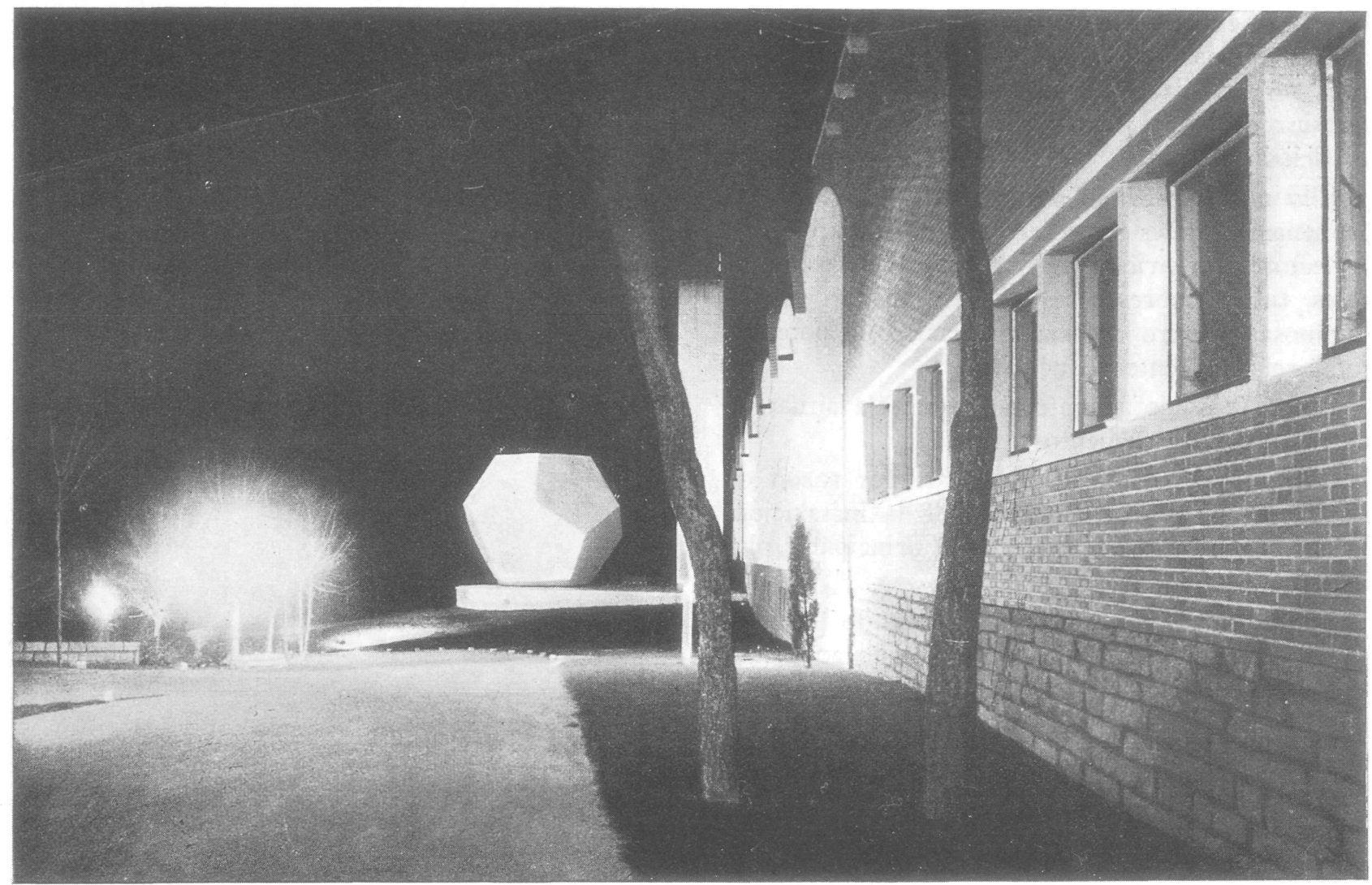

Fig. 8
I NSTITUTO
$T E C N \mid C O$
$D E$
L A
CONSTRUCCION
DE L
C EMENTO 
El resto de las dependencias, y de un modo particular las naves de estudios y laboratorios, presentan unas características de gran uniformidad que indican claramente la conveniencia de adoptar soluciones normalizadas. La modulación del edificio es, como veremos, un elementos decisivo en el estudio de la unidad-tipo de iluminación.

\section{III. - ALUMBRADO EXTERIOR}

La instalación de alumbrado exterior que en este momento existe ha sido proyectada, de acuerdo con las directrices recibidas, con finalidad utilitaria.

Quiere esto decir que no se trata de una iluminación decorativa o festiva, destinada fundamentalmente a proporcionar un espectáculo grandioso a los paseantes. Se trata, por el contrario, del conjunto de elementos precisos para hacer posible con comodidad el acceso nocturno a los edificios y su vigilancia.

Lo anterior no excluye el que, de acuerdo con las ideas expresadas al principio, se haya intentado acamodar la instalación a las construcciones para no inutilizar durante la noche el esfuerzo creador de las personas que han proyectado los edificios.

Ello no\$ ha llevado a disponer una diversidad de elementos, tales como farolas con lámparas $\mathrm{ML}$, reflectores de varios tipos y potencias, brazos murales, tubos fluorescentes de alta tensión y setas luminosas. dentro de una instalación de potencia total relativamente reducida.

En un croquis adjunto se detalla la situación de los distintos elementos (fig. I).

Del conjunto debemos destacar, por razón de su importancia relativa dentro de la instalación, la parte correspondiente al acceso principal y plaza de los sietes.

El camino de entrada está alumbrado por medio de farolas de diseño especial que soportan, cada una, una lámpara del tipo ML.

La altura de las farolas, inferior a los cuatro metros, y la relación entre ésta y la separación horizontal, superior a seis, declaran que se trata de un alumbrado de tipo jardín o parque.

Las farolas, diseñadas por los arquitectos de

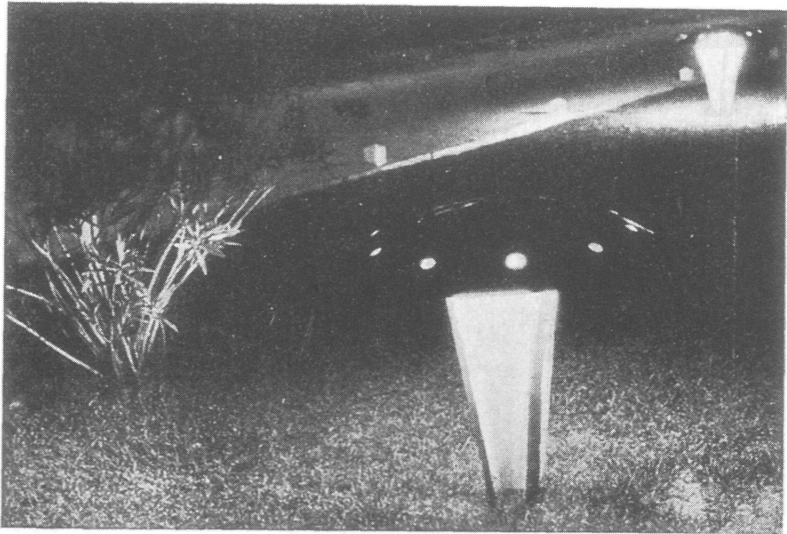

Fig. 9

la obra, son de silueta estilizada y están situadas a un solo lato del camino. Las figuras números 2 y 3 representan, respectivamente, el aspecto nocturno y diurno de las farolas.

La razón por la que se han dispuesto en el lado interior de la curva, en contra de lo que es costumbre en el alumbrado de vías de circulación. ha sido dob!e:

En primer lugar, colocadas a lo largo del bordillo exterior de la curra hubiesen mejorado, ciertamente, la eficacia del alumbrado pero, como contrapartida, entorpecerían la visión del depósito dodecaédrico, la chimenea y las naves de talleres. Estando éstos entre los elementos arquitectónicos más característicos del conjunto y considerando las razones de nuestro preámbulo, se estimó oportuno sacrificar el funcionamiento de

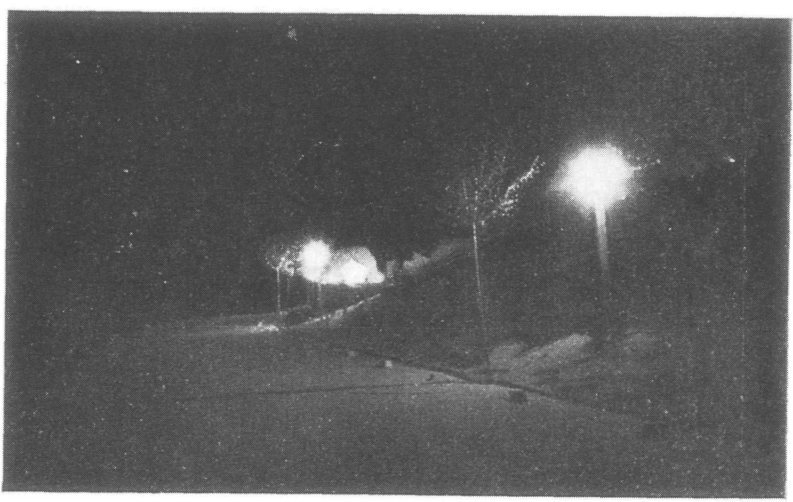

Fig. 10 


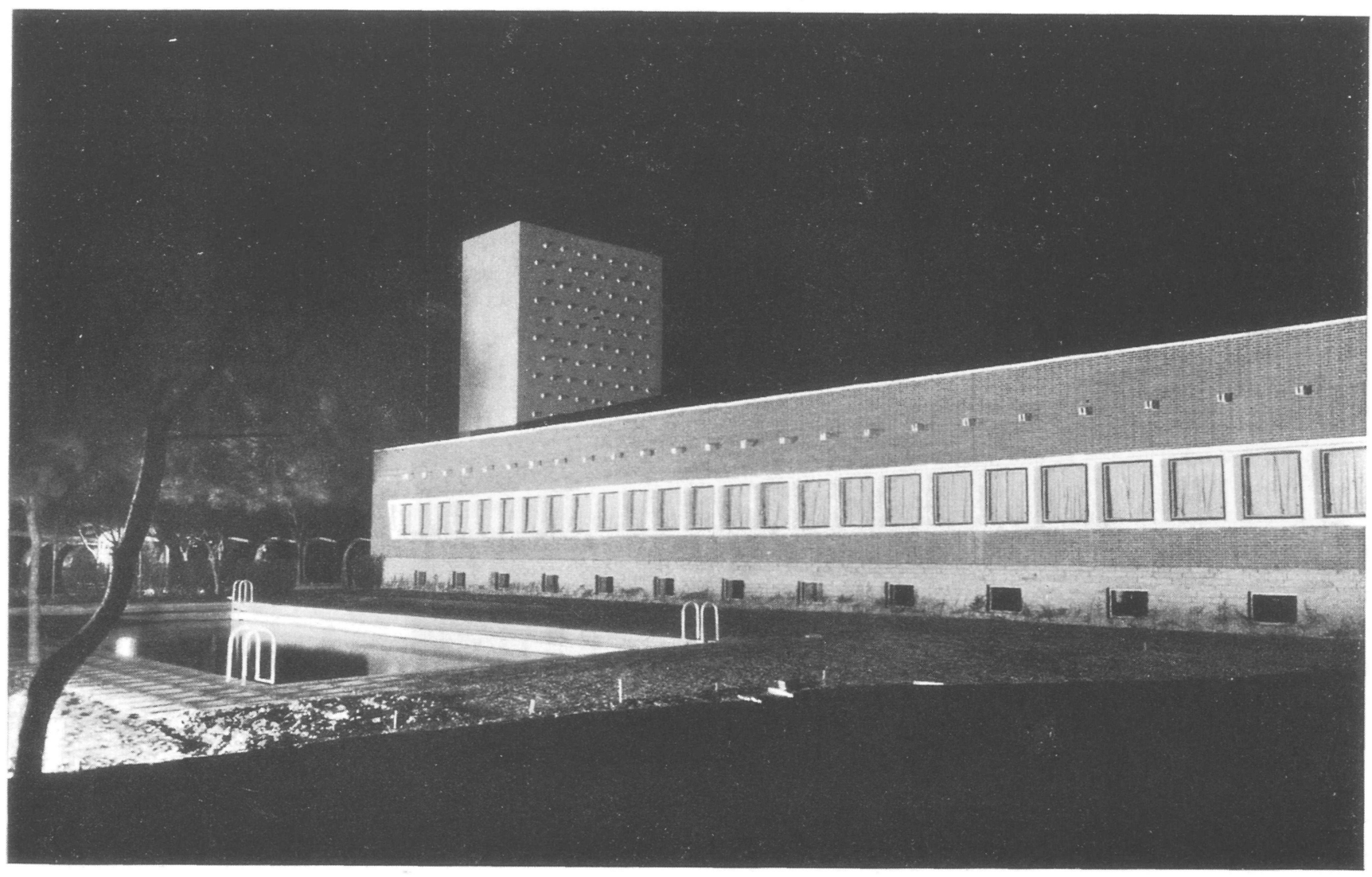

Fig. 11

las farolas para no desviar la atención de los observadores y conservar, de noche, la composición arquitectónica.

La segunda razón relaciona el emplazamiento de las farolas con el resto de los elementos luminosos del acceso y puede expresarse así:

Tanto el dodecaedro como la chimenea y el frente de las naves presentan superficies sumamente claras y de elevado rendimiento fotométrico en la reflexión.

Esta circunstancia permite complementar la visibilidad producida por las farolas por medio de luz reflejada sobre los paramentos claros.

Para ello se han dispuesto dos baterías de reflectores a ambos lados del depósito de carbón. De este modo la luz que racilita la circulación sirve, simultáneamente, para hacer visible la arquitectura (figuras núms. 5 y 6).

La figura número 6 muestra el aspecto nocturno del dodecaedro y la chimenea. En ello se aprecia la situación de una de las baterías de reflectores. La figura está tomada de tal modo que reproduce la impresión visual recibida por una persona que se aproxima a los edificios a lo largo de la ría de acceso.

La figura 8 corresponde a una persona que se disponga a abandonar el Instituto, saliendo en automóvil. Obsérvese el efecto perturbador de las farolas e imagínese el entorpecimiento visual que representarían situadas a lo largo del borde exterior del camino.

La vía de acceso desemboca en la plaza de los sietes. Aquí nos encontramos con una gran explanada en cuyo centro existe una zona de césped $y$ dos islotes, igualmente verdes, para encauzar la circulación.

Los elementos arquitectónicos más notables son :

Ln cubierta de sombra para el aparcamiento de a:tomóviles, cuyos soportes dan nombre a la pliza. 


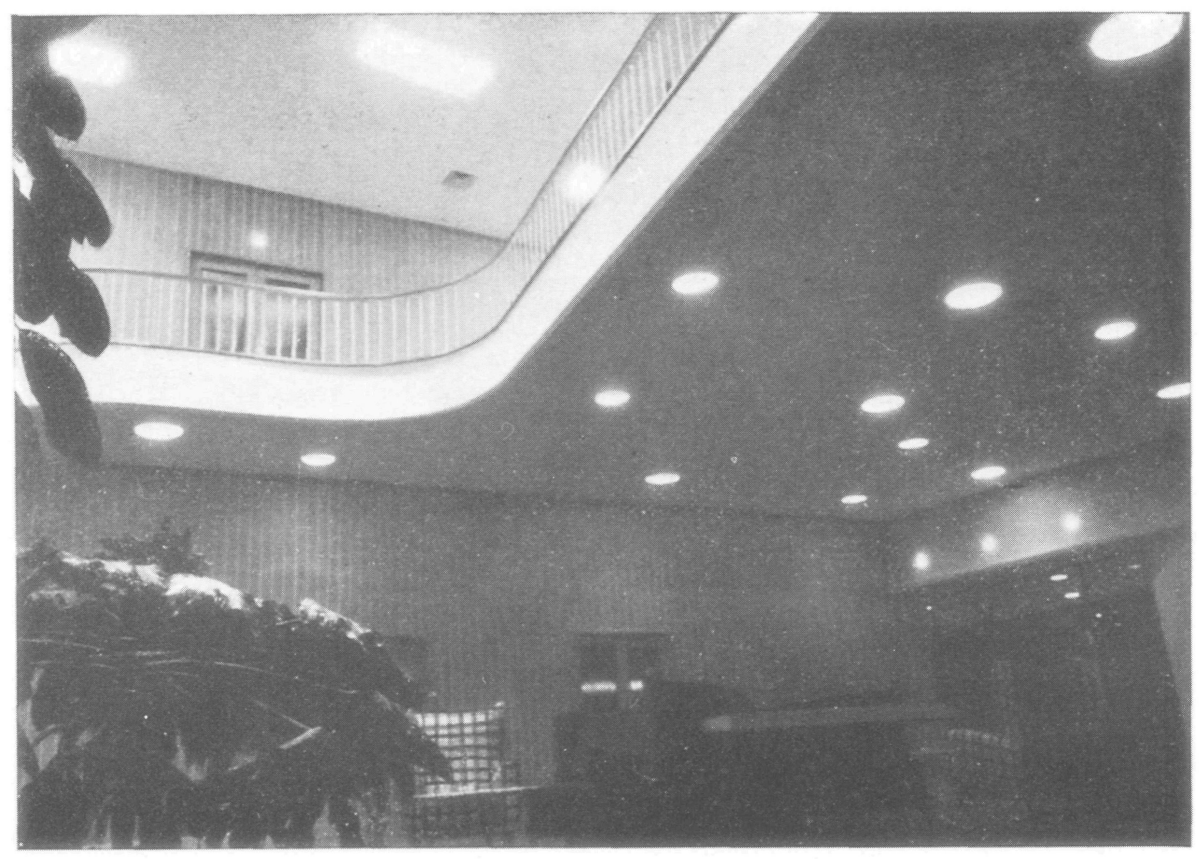

Fig. 12

El paramento claro de la sala de conferencias, que destaca de las restantes superficies de ladrillo.

La nave de estudios, con su doble hilera de ventanas.

La zona verde central.

Estos elementos han servido para diseñar el alumbrado, del modo que a continuación comentamos.

La cubierta sobre el aparcamiento, de línea esbelta, ha sido rematada por una línea luminosa constituída por un tubo fluorescente de alta tensión de color blanco.

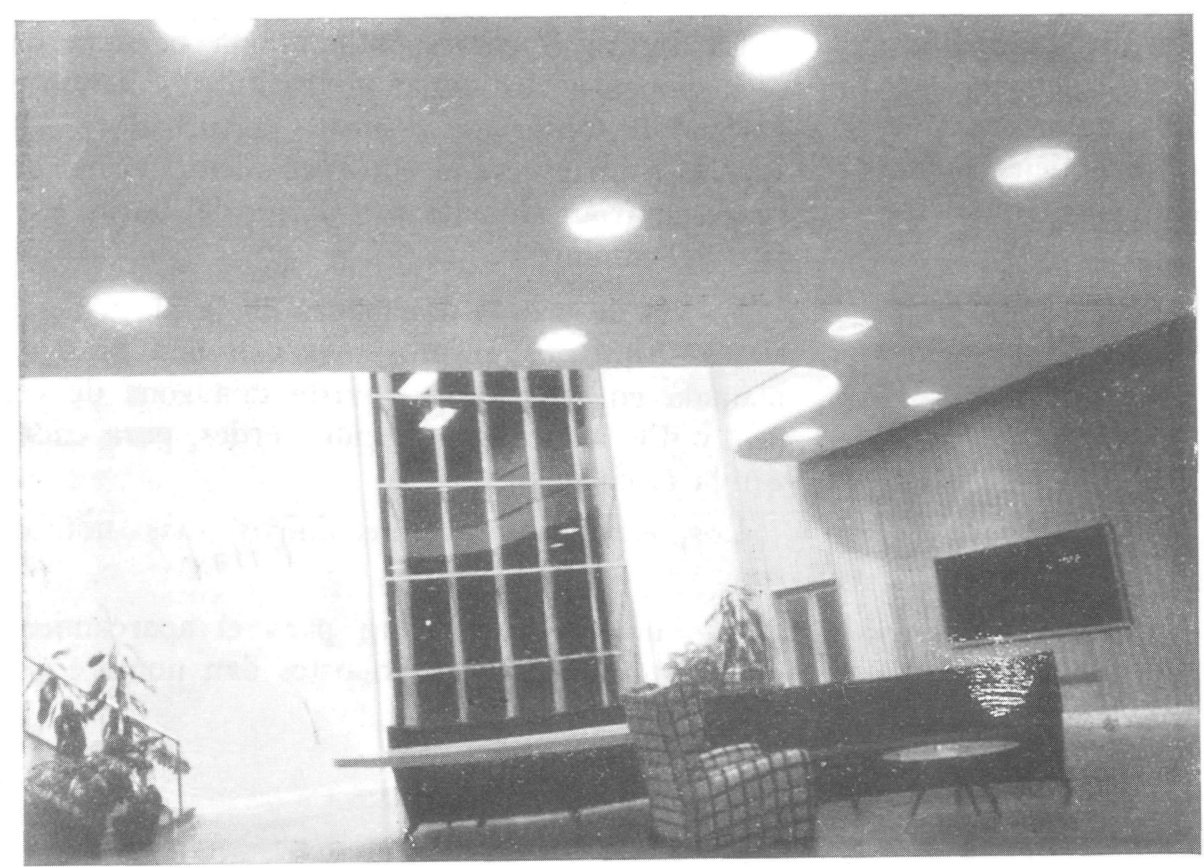




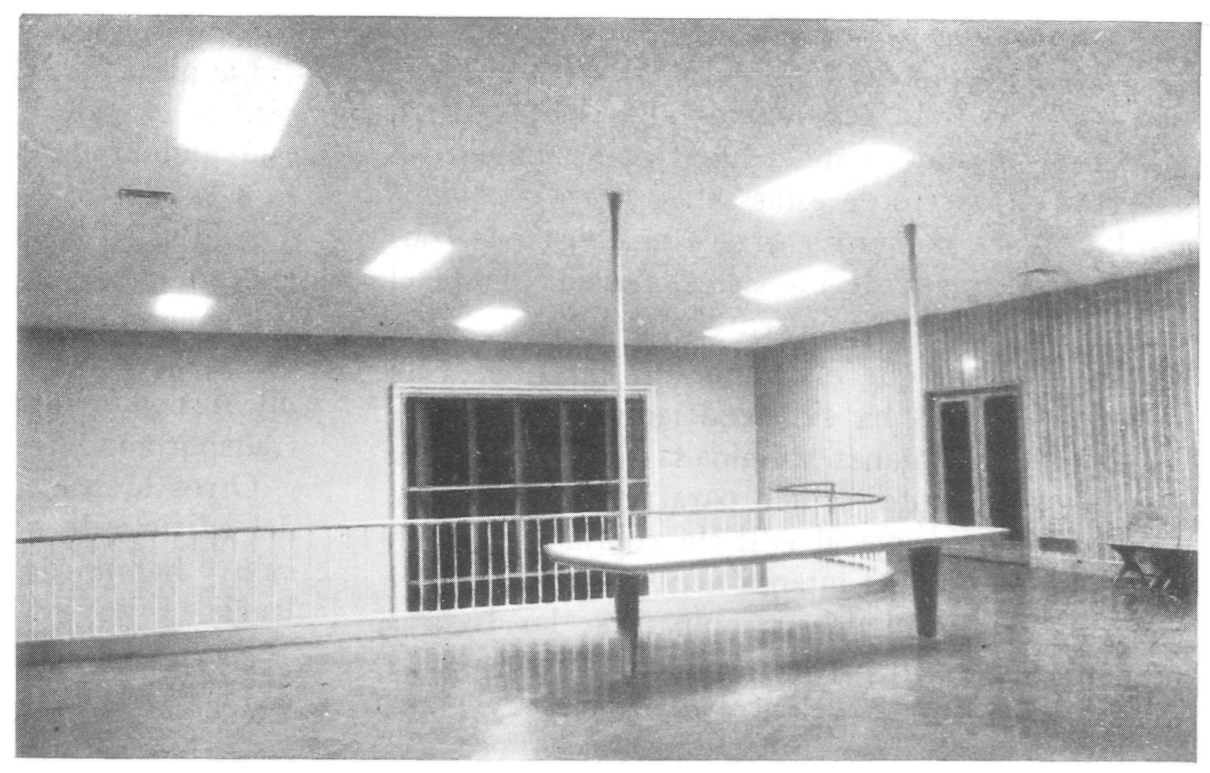

Fig 14

Esta línea horizontal sirve, además de contribuir al alumbrado general, para delimitar la zona de aparcamiento y para dar una sensación de amplitud.

El paramento claro de la sala de conferencias se ha alumbrado por medio de dos reflectores. De este modo se conserva el aspecto diurno, se cierra la perspectiva y se aprovechan las excelentes condiciones fotométricas de tal superficie para el alumbrado general.

La zona verde central se ha dotado de cuatro setas luminosas de sesenta centímetros de altura, de base de hormigón, construídas en el taller

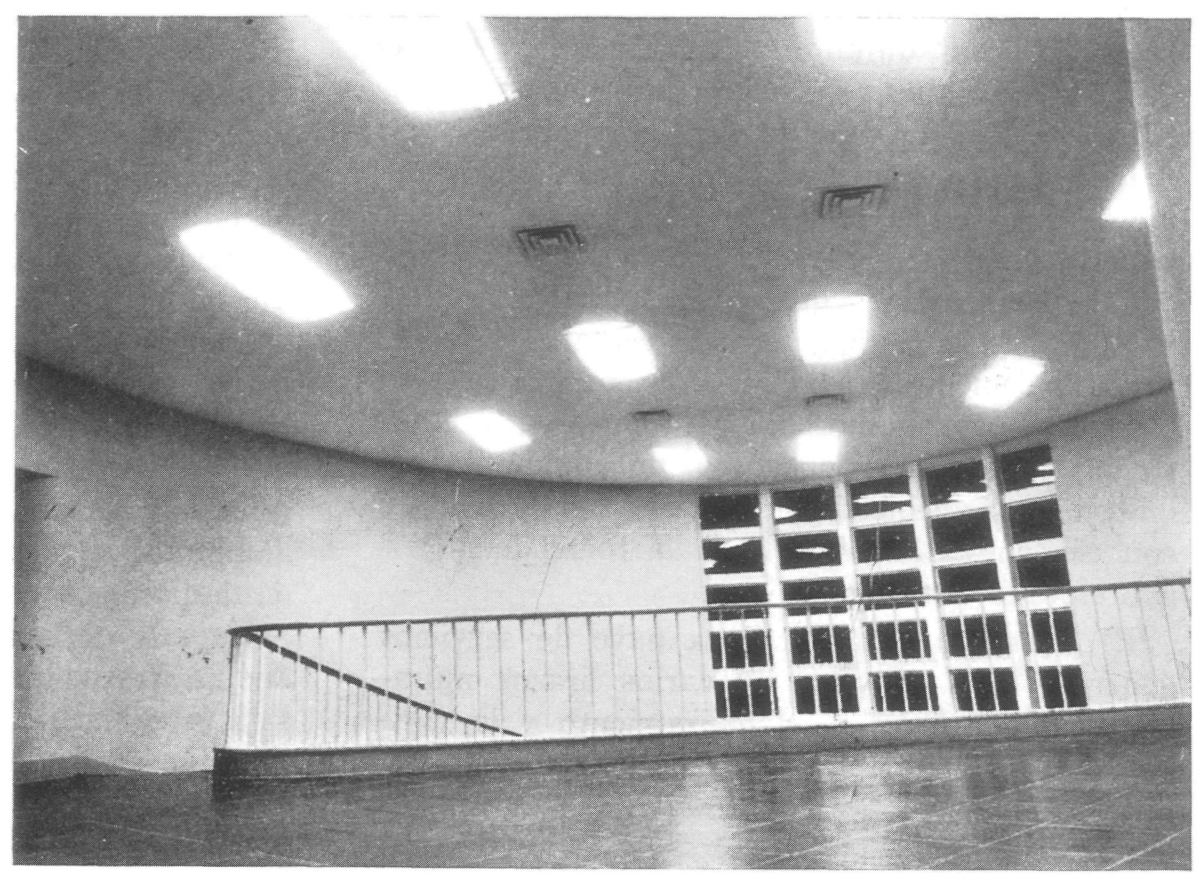


de prefabricación del Instituto, y cabeza de chapa pintada al duco. Cada una de ellas aloja cuatro lámparas incandescentes. La figura número 8 muestra el aspecto de estas setas.

La figura número 7 ofrece una vista de conjunto de la plaza de los sietes, tomada desde el mismo lugar que la número 8.

Se observará que el alumbrado exterior de la nave de estudios ha sido confiado fundamentalmente a las ventanas iluminadas exteriormente. Este procedimiento, propio para alzados austeros, pone de relieve que el edificio cobija locales en los que se desarrolla intensa actividad. Para apreciar esta sensación deben compararse las figuras números $4 \mathrm{y} 5$, que corresponden a los aspectos diurnos y nocturno del acceso principal y plaza de los sietes. Nótese como, durante la noche, la nare de estudios cobra un aspecto de gran vitalidad.

De la comparación de estas dos figuras puede deducirse hasta qué punto se ha logrado respetar la composición arquitectónica, aprovechando sus propios elementos parą el alumbrado artificial

El resto de la instalación de alumbrado exterior, por corresponder a zonas menos transitadas, ha sido concebido fundamentalmente con la finalidad de facilitar la labor de vigilancia nocturna.

El patio de la alberca, situado entre las naves de estudios, servicios generales y laboratorios, es alumbrado por medio de dos reflectores colocados, respectivamente, sobre la nave de Dirección y sobre la de laboratorios, junto a la torre del depósito elevado.

No se han dispuesto farolas ni brazos murales por no alterar, durante el día, el aspecto sereno de este patio, que no se vería beneficiado por la adición de ningún aparato de alumbrado.

La zona de deportes ha sido alumbrada por medio de dos baterías de reflectores dirigidas hacia la fachada meridional de la nave de laboratorios.

Esta iluminación, que puede permitir el uso nocturno de la piscina, tiene la ventaja de facilitar notablemente la vigilancia. Al mismo tiempo, puede utilizarse como base para instalar, si alguna vez se considera oportino, un alumbrado arquitectónico visible desde la calle, completándola con elementos dirigidos hacia la torre del depósito elevado para agua.

E1 alzado a Levante de la nave de servicios generales está provisto de varios brazos murales, cuya finalidad es el balizamiento y la vigilancia.
La plaza que forman la nave de ensayos mecánicos y talleres $\mathrm{y}$ el edificio de hormigones está alumbrada por medio de una farola, idént1ca a las del acceso principal, situada aproxima lamente en su centro y entre los árboles. Además, existen puntos de luz en los porches de talleres y hormigones, que habrán de facilitar las operaciones de carga y descarga de materiales, herramientas y maquinaria de los camiones que las transporten.

Otros brazos murales, situados en las esquinas del edificio de hormigones, completan la instalación y habrán de servir para balizamiento y vigilancia.

\section{IV. - ALUMBRADO INTERIOR}

De un modo general, y con la finalidad de sistematizar el estudio de la instalación de alumbrado interior, dividíamos las dependencias del Instituto en tres tipos:

a) Despachos y oficinas.

b) Nares de estudios y laboratorios.

c) Servicios, cocinas, vestuarios, aseos, dormitorios, etc.

Además, y como elementos fuera de serie, conside:amos la nave de Dirección, conferencias, hall principal $\mathrm{y}$ de personal y el comedor.

Estos puntos singulares planteaban un problema que encaja mejor en el ámbito de la decoración que en el de la técnica del alumbrado. Por tal razón fueron estudiados de un modo especial por la Sección de Arquitectura, que tomó en ellos rina parte muy importante, limitándose nuestra colaboración a indicar la potencia que debía ser instalada.

Dentro de la nave de Dirección se instaló alumbrado incandescente en secretaría, sala de visitas y coloquios, así como en los servicios. El despacho del director está provisto de alumbrado incandescente y fluorescente. La oficina aneja dispone de alumbrado fluorescente análogo al de las zonas de despachos y oficinas.

La sala de conferencias, con sus dependencias anejas, forma un conjunto independiente. En la sala propiamente dicha se ha instalado una combinación de luz incandescente, en reflectores em- 
potrados en el techo con rejillas difusoras circulales, y fluorescente indirecto.

En el guardarropa y sala de proyección, fluorescente. En los pasillos de acceso, aseos, etc., alumbrado incandescente en globos de vidrio esmerilado y apliques.

El hall principal está alumbrado, en su nivel inferior, por medio de reflectores incandescentes empotrados $y$ provistos de rejillas difusoras. El piso superior dispone de equipos fluorescentes triples empotrados y con rejilla metálica. Las figuras I2, I3 y I4 se refieren a este lugar.

El hall de personal está resuelto de un modo análogo al anterior, pudiéndose apreciar en la figura número I $5_{5}$.

La entrada de personal está alumbrada por medio de apliques metálicos con lámparas incandescentes y reflectores circulares empotrados.

En el comedor de empleados se han instalado apliques, iguales a los anteriores, en las columnas centrales.

Hecha la anterior y brevísima descripción de los puntos singulares, pasemos a considerar el grueso de la instalación de alumbrado interior:

Las características fundamentales o generales que se han querido conseguir para la instalación de que nos estamos ocupando son la utilidad y la economía.

La utilidad incluve la calidad del alumbrado que, en cada lugar, debe ser adecuado a la tarea risual a realizar.

La utilidad nos marcó los niveles luminosos que habían de servir de base a los cálculos. El criterio aplicado fué el siguiente:

\begin{tabular}{|c|c|}
\hline ión & \\
\hline las y despa & 200 \\
\hline e visitas y coloc & IOO \\
\hline$=$ & $5^{\circ}$ \\
\hline Galerías & 25 \\
\hline
\end{tabular}

La economía fué una de las consideraciones que mayor influencia turo en la búsqueda de la solución más conveniente.

Esta cualidad había de incluir no sólo los gastos de primer establecimiento, sino también el gasto anual de consumo de energía, reposición de elementos, limpieza y reparaciones, etc.

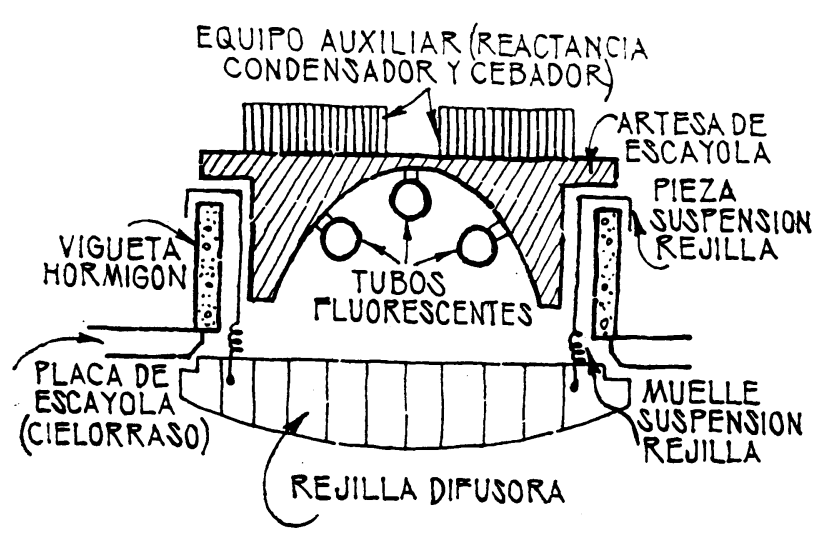

Fig. 16

Las tres primeras consideraciones admitian ser expresadas por medio de números, lo que nos llevó a la estimación del coste en pesetas de un lumen durante un año para una instalación incândescente y para otra fluorescente.

Se partió del coste de adquisición de los equipos de alumbrado, del precio del kilovatio-hora. de la rida media de las lámparas y de su rendimiento luminoso, estableciéndose el coste de un lumen durante un año.

En estas condiciones llegamos a las siguientes cifras:

Incandescente: O, I49 ptas./lumen año.

Fluorescente: 0.092 ptas. /lumen año.

Que corresponderían a una instalación que funcionase ininterrumpidamente.

Como este caso no coincide con la realidad, hicimos intervenir el número de horas de funcionamiento anual, partiendo de los datos anteriores.

De este modo llegamos a la conclusión de que, por debajo de 300 horas (en números redondos) de funcionamiento anual, era más barato el sistema incandescente. Por encima de esta cifra la instalación fluorescente of recía rentajas económicas.

Como consecuencia de lo anterior, adoptamos en líneas generales la modalidad fluorescente en aquella parte de la instalación cuya utilización debía preverse por encima de las 300 horas al año. El resto sería incandescente.

Tamién consideramos la influencia de la longitud de los períodos de encendido en la vida de 

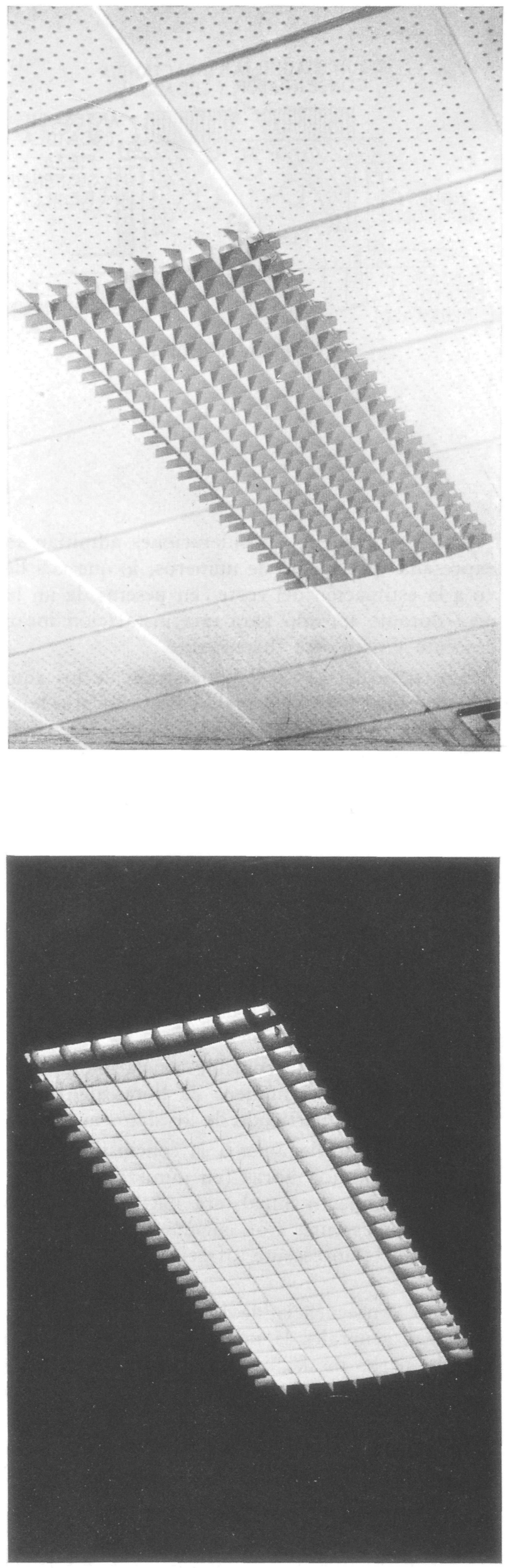

Fig. 17

las lámparas fluorescentes, pues es conocida la perjudicial influencia de los frecuentes apagados $\mathrm{y}$ encendidos.

Con los anteriores criterios, se procedió a estimar la potencia precisa en las distintas dependencias. Para ello se utilizó el sistema de los niveles medios o método de los flujos, estimando los coeficientes de utilización por el método del holandés H. J. Zijl, de la oficina técnica de Philips en Eindhoven.

Dimensionada, en principio la instalación, se procedió al estudio de la forma en que debía realizarse prácticamente.

Aquí hubieron de tenerse en cuenta una serie de factores, de entre los cuales son los más notables los siguientes:

Normalización de las soluciones.

Facilidad de montaje.

Facilidad de reposición de lámparas.

Calidad del alumbrado: efecto estroboscópico y deslumbramiento.

Adaptación de los aparatos de luz a las modalidades constructivas y arquitectónicas del edificio, etc., etc.

Los anteriores puntos de vista no son totalmente independientes entre sí, sino que, por el contrario, se relacionan unos con otros, llegando a interferir en determinados aspectos. Ello determinó la conveniencia de adoptar una solución de compromiso, resultado de una serie de consideraciones cuyo detalle omitimos.

Unicamente nos referimos a la influencia de las características constructivas y arquitectónicas de los edificios.

La composición, en planta, del nuevo Instituto está realizada sobre la base de un módulo o célula elemental de I,60 X I,60 m. En los supuestos de distribución interior de las diferentes naves entraba la posibilidad de que, en cualquier momento, fuese posible modificar la situación de los tabiques con el fin de adaptar las disponibilidades de espacio a las, posiblemente, variables necesidades del trabajo.

Esta circunstancia debía ser recogida en el alumbrado interior. Era, pues, preciso proyectar una instalación flexible, que pudiese acomodarse a las previstas variaciones de distribución. De las soluciones consideradas, pareció la más acertada 
aquélla que consistía en aplicar la modulación a los puntos de luz. Es decir, en distribuirlos regularmente sobre la cuadrícula de i,6o $\times$ i,6o.

Faltaba entonces por resolver la cuestión de modificar la potencia de los equipos, de acuerdo con la variación previsible en las necesidades, va que un lugar destinado a laboratorio podría, eventualmente, transformarse el día de mañana, en sala de risitas o viceversa.

Con todo lo anterior, formamos cuatro unidades-tipo de alumbrado, correspondientes a los niveles medios de 50, I00, 200 y 300 lux respectiramente, de que hablamos más arriba.

Determinamos; a continuación, las potencias y separaciones correspondientes a cada uno de ellos. que resultaron ser, aproximadamente:

Zona de 300 lux $\ldots . . . . .4$ 40 vatios por módulo. Zona de 200 lux ........ 30 ratios por módulo. Zona de 100 lux $\ldots . . . . . .20$ vatios por módulo. Zona de 50 lux ........ io ratios por módulo.

Fstas cifras se obtuvieron del análisis de los resultados obtenidos por el sistema $\mathrm{Zijl}$.

Considerando, después, que no era probable que una zona de 50 lux (pasillo) pasase a ser despacho ni laboratorio, se estudió el problema de diseñar una unidad-tipo que pudiese ser transformada fácilmente de modo que sirviese indistintamente para zonas de I0O, 200 ó 300 lux.

E1 resultado de toto ello fué la solución que se detalla en el esquema de la figura i6.

Sobre las riguetas que soportan el cielorraso de placas de escayola, se colocan las piezas de suspensión de la rejilla, construídas en chapa doblada y de las que penden los muelles terminados en un gancho.

Sobre las riguetas se disponen después las artesas de escayola, construídas en el taller de prefabricación del Instituto, y a las que, previamente, les ha sido colocada la instalación eléctrica completa (portalámparas, reactancias, cebadores y condensadores).

Las dos operaciones anteriores se realizan desde las entre!lantas visitables de que está provisto el edificio.

Una rez colocada la artesa, se acoplan los tubos a los portalámparas y, a continuación, se suspende la rejilla de los cuatro muelles que la mantienen aplicada contra el cielorraso.
Estas dos últimas operaciones se hacen desde abajo.

La reposición de lámparas se realiza con suma facilidad, ya que basta quitar la rejilla tirando ligeramente de los muelles de suspensión, con lo que los tubos quedan al alcance de la mano. Lo mismo ocurre con la limpieza.

La transformación de una unidad de un tipo en una de otro se realiza simplemente con suprimir o añadir alguna lámpara, ya que las unidades se han dispuesto a separaciones tales que los tres niveles luminosos corresponden a equipos sencillos, dobles o triples.

En caso de arería, la reparación se hace sustituyendo la artesa completa por otra de las que se conservan de repuesto.

La operación es rápida, puesto que las artesas están únicamente apoyadas sobre las viguetas.

La supresión de un punto de luz se consigue, también, con facilidad, ya que la abertura inferior es precisamente la correspondiente a tres placas de cielorraso $(\mathrm{I}, 2 \mathrm{O} \times 0,40 \mathrm{~m}$.), bastando para ello quitar la rejilla $y$ la artesa y colocar tres placas.

Con la unidad-tipo que acabamos de describir se consiguió sistematizar notablemente el trabajo de montaje, "prefabricando" los equipos en taller y limitándose la labor "in situ" a colocar las artesas y rejillas y a empalmar con las redes de distribución de energía.

El alumbrado de las naves de estudios y laboratorios se ha realizado íntegramente con unidades-tipo como la descrita.

En los pasillos se han dispuesto unidades análogas, cuya única particularidad es la sustitución de la rejilla por vidrio estriado.

En las zonas industriales se ha seguido el mismo criterio y se han aplicado análogas unidades-tipo. En estos casos, sin embargo, se ha prescindido de la rejilla difusora en beneficio de un mejor rendimiento luminoso y aceptando el deslumbramiento algo mayor por tratarse de lugares en los que la tarea visual está más concentrada y donde la decoración desempeña un papel secundario.

En los lugares en que el cielorraso no está constituído por placas de escayola, se ha sustituído la artesa por una armadura metálica adaptable para equipos sencillos, dobles o triples, conservándose, por lo tanto, la flexibilidad.

Las figuras números I7 y $\mathrm{I} 8$ reproducen el aspecto de las unidades-tipo durante el día y la 
noche. La figura I9 corresponde a uno de los pasillos de la nave de estudios.

El problema del efecto estroboscópico, especialmente molesto en salas de mecanografía y talleres, se resolvió por medio del montaje trifásico de los equipos fluorescentes.

Con lo anterior nos hemos referido a las naves industriales, a los despachos y a las oficinas. Nos resta por hablar de los servicios, cocinas, vestuarios, etc., etc.

Esta parte de la instalación planteaba cuestiones menos comprometidas y de menor trascendencia. Aquí no era preciso imaginar soluciones especiales, puesto que correspondía a casos normales en la práctica del alumbrado artificial.

Aplicamos el criterio de las horas previsibles de funcionamiento para adoptar solución incandescente o fluorescente, decidiéndonos por el primero en la cocina, oficio, salas de lavado y plancha, pasillos y otros.

Las restantes dependencias (aseos, cabinas te- lefónicas, vestuarios...) se alumbraron por medio de lámparas incandescentes, de potencias comprendidas entre 25 y 200 vatios, instaladas en apliques murales o globos esmerilados.

Análoga solución se adoptó para las galerias de servicios del sótano y para la instalación de alumbrado supletorio o de socorro. Esta última, destinada a la vigilancia nocturna interior y a la evitación de oscuridad completa en caso de avería, está constituída por una serie de lámparas de 25 w. situadas de tal modo que permiten recorrer todas las dependencias del Instituto sin encender el alumbrado normal.

La figura número I9 muestra encendidos algunos puntos de luz supletoria situados sobre las puertas.

La alimentación eléctrica del alumbrado supletorio está concebida de forma que es posible encenderlo con los medios propios del edificio, en cuanto se produce alguna interrupción en la instalación general.

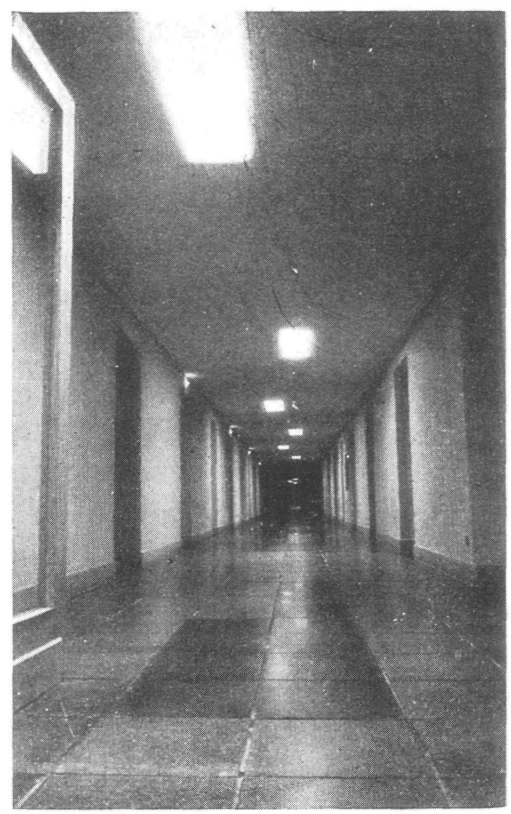

